\title{
A Critical Appraisal of Circumferential Resection Margins in Esophageal Carcinoma
}

\author{
Bareld B. Pultrum, MD ${ }^{1}$, Judith Honing, MD ${ }^{1}$, Justin K. Smit, MD ${ }^{1}$, Hendrik M. van Dullemen, MD ${ }^{2}$, \\ Gooitzen M. van Dam, PhD ${ }^{1}$, Henk Groen, PhD $^{4}$, Harry Hollema, PhD $^{3}$, and John Th. M. Plukker, PhD $^{1}$ \\ ${ }^{1}$ Department of Surgery, Division of Surgical Oncology, University Medical Center Groningen, University of Groningen, \\ Groningen, The Netherlands; ${ }^{2}$ Department of Gastroenterology, University Medical Center Groningen, University of \\ Groningen, Groningen, The Netherlands; ${ }^{3}$ Department of Pathology and Laboratory Medicine, University Medical Center \\ Groningen, University of Groningen, Groningen, The Netherlands; ${ }^{4}$ Department of Epidemiology, University Medical \\ Center Groningen, University of Groningen, Groningen, The Netherlands
}

\begin{abstract}
Background. In esophageal cancer, circumferential resection margins (CRMs) are considered to be of relevant prognostic value, but a reliable definition of tumor-free CRM is still unclear. The aim of this study was to appraise the clinical prognostic value of microscopic CRM involvement and to determine the optimal limit of CRM.

Methods. To define the optimal tumor-free CRM we included 98 consecutive patients who underwent extended esophagectomy with microscopic tumor-free resection margins (R0) between 1997 and 2006. CRMs were measured in tenths of millimeters with inked lateral margins. Outcome of patients with CRM involvement was compared with a statistically comparable control group of 21 patients with microscopic positive resection margins (R1).

Results. A cutoff point of CRM at $\leq 1.0 \mathrm{~mm}$ and $>1.0 \mathrm{~mm}$ appeared to be an adequate marker for survival and prognosis (both $P<0.001$ ). The outcome in patients with CRMs $\leq 1.0$ and $>0 \mathrm{~mm}$ was equal to that in patients with CRM of $0 \mathrm{~mm}(P=0.43)$. CRM involvement was an independent prognostic factor for both recurrent disease $(P=0.001)$ and survival $(P<0.001)$. Survival of patients
\end{abstract}

Presented at the Gastrointestinal Cancers Symposium 2009 in San Francisco and at the 62nd Cancer Symposium 2009 of the Society of Surgical Oncology in Phoenix.

(C) The Author(s) 2009. This article is published with open access at Springerlink.com

First Received: 16 June 2009;

Published Online: 19 November 2009

J. Th. M. Plukker, PhD

e-mail: j.th.plukker@chir.umcg.nl with positive CRMs $(\leq 1 \mathrm{~mm})$ did not significantly differ from patients with an $\mathrm{R} 1$ resection $(P=0.12)$.

Conclusion. Involvement of the circumferential resection margins is an independent prognostic factor for recurrent disease and survival in esophageal cancer. The optimal limit for a positive CRM is $\leq 1 \mathrm{~mm}$ and for a free CRM is $>1.0 \mathrm{~mm}$. Patients with unfavorable CRM should be approached as patients with R1 resection with corresponding outcome.

The rising incidence and poor prognosis of esophageal adenocarcinoma have intensified research efforts to find better staging modalities, early detection, and treatment methods. ${ }^{1,2}$ Surgery, as the only curative option, provides better outcome with local tumor control than nonsurgical treatment. ${ }^{3,4}$ Histological examination of surgical resection margins is a standard procedure to determine the radicality (denominated as $\mathrm{R}$ ) of resection. It is crucial that resection margins are microscopically free of tumor (R0) to prevent local recurrences and avoid consequently poor survival. ${ }^{5,6}$

In rectal cancer, involvement of circumferential resection margins (CRMs) is regarded as a highly significant predictor of local recurrence, prognosis, and survival. ${ }^{7-10}$ This led to an alteration of practice, with recommendations for preoperative (chemo)radiotherapy with complete resection of the whole mesorectum in order to reduce the rate of CRM involvement. ${ }^{11}$

The role of CRMs in predicting local recurrence in esophageal carcinoma was first described by Sagar et al. ${ }^{12}$ Subsequently, the role of CRMs in esophageal cancer has been investigated during the past decade. In contrast with the stomach, small bowel, and colon, the esophagus lacks a serosal layer. Hence, tumor expansion encounters only a 
few barriers before surrounding structures are invaded. The involvement of CRMs depends upon a combination of tumor location and extension (ingrowth), variable esophageal wall thickness, and surgical technique. Therefore, CRMs could serve as a requirement for better local treatment and, similar to in colorectal surgery, as a useful quality indicator of surgery. ${ }^{7,9}$ Besides the presence of nodal metastases, lymph vascular space involvement, and intramural metastases, CRM involvement predicts poor prognosis. ${ }^{13}$ Some studies showed that microscopic tumor within $1 \mathrm{~mm}$ of the inked circumferential margin, which would be regarded as a potentially curative resection (R0), doubles the risk of mortality in the short to medium term (3-year survival). ${ }^{12,14}$ However, there is a lack of conclusive clinical data for a fixed definition of the optimal limit of tumor-free CRMs in esophageal cancer. Many centers still do not take into account CRMs as a routinely performed item. Furthermore, the literature about the clinical value of CRMs in esophageal cancer is sparse. Especially with current advanced staging procedures and selective use of neoadjuvant treatment, the clinical relevance of CRMs should be further examined.

Aim of this study was to appraise the clinical prognostic value of microscopic circumferential margin involvement in esophageal cancer and that of other histopathologic measurements influencing CRMs. CRM measurements were performed and we assessed whether it could be a relevant clinical prognostic factor regarding local recurrence and survival. Moreover, we determined the exact optimal limit of CRMs (in tenths of millimeters). Clinical recommendations and optimal treatment policy are also discussed.

\section{PATIENTS AND METHODS}

\section{Study Design}

This study was performed in 98 patients with cancer of the esophagus or gastroesophageal junction who underwent curative intended surgery from 1997 to 2006 in our tertiary referral university hospital. All patients underwent radical esophageal resection performed by the same surgical group, consisting of two experienced surgeons. For evaluation of the circumferential resection margins (CRMs) patients had a microscopic free surgical resection margin, an $\mathrm{R} 0$ resection, without evidence of distant metastases. Patients with microscopically positive resection margins, an $\mathrm{R} 1$ resection (defined as cases with presence of microscopic tumor cells within $1 \mathrm{~mm}$ of the proximal or distal surgical resection margins), were used as a control group for survival analysis. The patients in the latter group were treated by the same surgical group during the period 1992-
2006 ( $n=21$ patients). This group was statistically comparable to the study group (R0) regarding preoperative management, surgical procedures, and patient and tumor characteristics.

\section{Preoperative Management}

Staging was performed according to the 6th International Union against Cancer (UICC) tumor-nodemetastasis classification, which was preoperatively determined by conventional staging modalities, including endoscopic ultrasonography (EUS) in combination with fine-needle aspiration (FNA) and computed tomography (CT) of the neck, chest, and abdomen, with ultrasonography of the cervical region on indication. ${ }^{15}$ All patients with a T3-4 and/or N1 tumor were additionally staged by fluorodeoxyglucose positron emission tomography (FDGPET). PET/CT images were fused when indistinctness in staging occurred.

\section{Inclusion and Exclusion Criteria}

All included patients had a locally resectable, histologically proven cancer of the esophagus, without evidence of distant metastases (T1-4N0-1M0-1a). Patients with distant metastases (M1b) were excluded. The few cases with postoperative mortality (within 30 days or in-hospital mortality) were excluded. Patients who received neoadjuvant treatment, all within a randomized trial, were also excluded due to possible interfering factors.

\section{Surgical Procedure}

As a standard, we performed radical transthoracic subtotal esophageal resection through a left thoracolaparotomy with intrathoracic anastomoses in tumors of the distal esophagus and gastroesophageal junction or through a right thoracolaparotomy with a cervical anastomosis. Both were combined with a two-field lymphadenectomy of mediastinal and abdominal lymph nodes, including the nodes at the celiac trunk and along the upper border of the pancreas.

\section{Histological Examination}

All resected esophageal specimens were examined macroscopically for extension of the primary tumor and palpable lymph nodes while keeping the specimen intact. Subsequently, the outside of the specimen was painted with Indian ink according to the method of Quirke, for better microscopic assessment of the surgical and lateral margins. ${ }^{14}$ The specimens were then fixed in formalin for a minimum of $24 \mathrm{~h}$ before further evaluation. 
Transverse cross-sections of approximately $0.5 \mathrm{~cm}$ width of the specimen were prepared to determine macroscopically the minimal distance between the tumor and the nearest inked margin (Fig. 1a, b). For CRM determination, a minimum of two sections were taken of this area with minimal circumferential distance and stained with hematoxylin and eosin (H\&E) for microscopic examination (Fig. 2). The distance from the most lateral malignant cells to the nearest inked lateral margin (defined as the CRM) was measured microscopically in tenths of millimeters (Figs. 2 and 3).

Furthermore, all specimens were assessed for surgical resection margins ( $\mathrm{R}$ classification), lymph node (LN) involvement, and the following tumor characteristics: type and grade of tumor, pattern of growth (pushing or satellite growth), and types of invasion, plus lymph vascular space involvement $(\mathrm{LVSI}=$ lymphogenic and/or angiogenic growth) and perineural invasion.

\section{Follow-Up}

Relevant follow-up was obtained from the prospectively collected data. Patients were followed every 3 months for
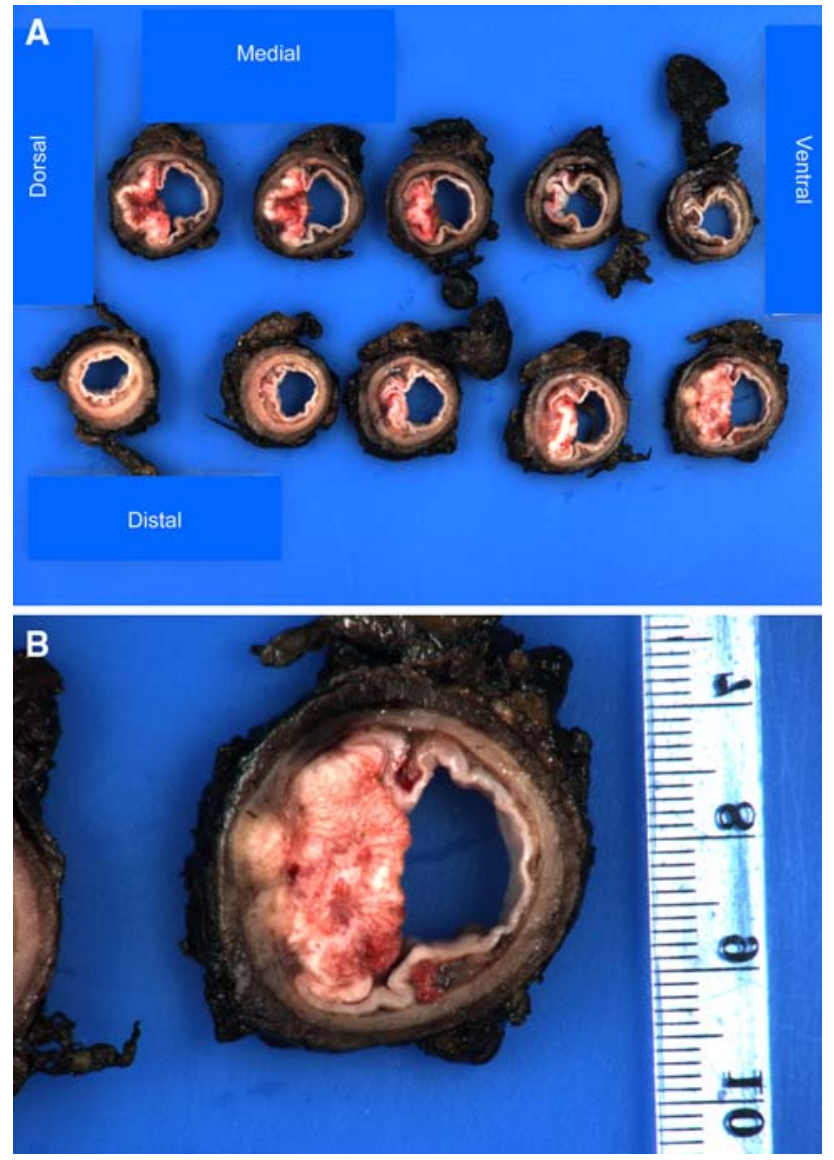

FIG. 1 a Cross-sections of a specimen through the tumor with 0.5cm-wide slices. b Cross-section with macroscopically minimal distance between the tumor and the nearest inked margin

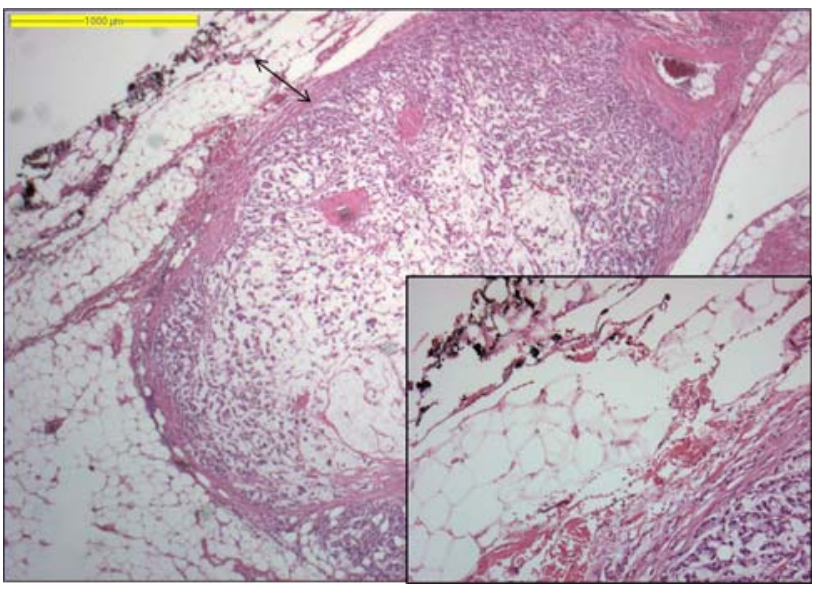

FIG. 2 Microscopic example of CRM measurement, from most lateral tumor cells to the inked outer margins; an enlargement of the margin is shown in the inset
A

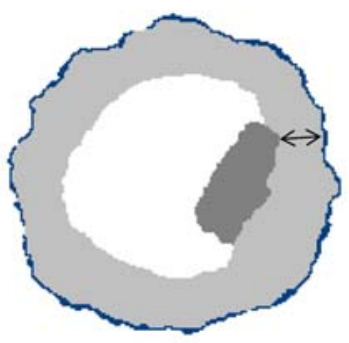

C

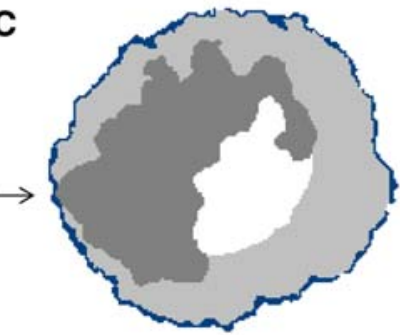

Esophageal wall

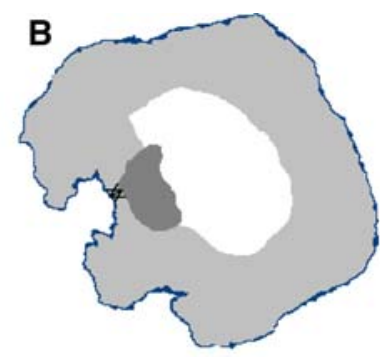

D

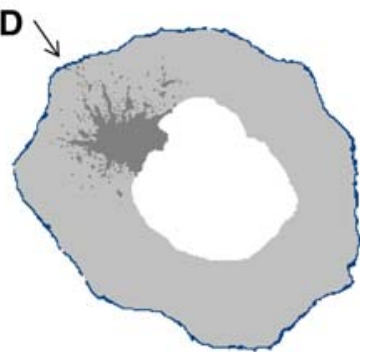

Tumor
Inked lateral margin
FIG. 3 Schematic representation of four possible microscopic circumferential resection margins (CRM) measurements in different esophageal cross-sections: a tumor with free CRM, measurement performed in tenths of millimeters; b small tumor with narrow free CRM, damage to the esophageal wall caused by surgical manipulation; c large tumor, directly growing into the CRM; d tumor with satellite (spray) growth into the CRM with few malignant cells

the first postoperative year, every 6 months for the next year, and then annually for 10 years. Any recurrent disease occurring within 3 months after operation was defined as persisting disease. All patients were followed, with a minimum of 2 years after surgery or until death. No patients were lost to follow-up. Survival was measured in months; cancer-related death was scored as an event; death of any other cause was scored as end of follow-up. Recurrence was defined as tumor regrowth, determined by 
any cytologic or histologic proof, unequivocal radiologic suspicion (CT, MRI, PET, bone scan, and ultrasonography), and/or obvious clinical manifestations. Survival of patients with determined tumor-free CRMs (CRM-) and tumor-positive CRMs (CRM+) were compared with survival of the patients in the control group (R1).

A division was made in number of positive lymph nodes (histology-proven positive lymph nodes) at $>4$, and in lymph node ratio (positive lymph nodes count/examined lymph nodes count) at $>0.20$; recently it appeared that these factors are important independent prognostic indicators. ${ }^{16,17}$ Length of tumor was derived from EUS measurement. On the grounds of previously published data, length was divided into two groups: $\leq 5 \mathrm{~cm}$ and $>5 \mathrm{~cm}^{18}$

\section{Patient and Tumor Characteristics}

The study population consisted of 98 consecutive patients: 76 males (77.6\%) and 22 females (22.4\%). Mean age was 64 years with a range of 41-81 years (Table 1). The overall R0 percentage of all surgically treated patients in our center was $89 \%$. Consequently, $11 \%$ of the resections were assessed as R1 resection.

Most tumors were adenocarcinomas $(n=75,76.5 \%)$ of the gastroesophageal junction $(n=44,44.9 \%)$ or distal esophagus $(n=43,43.9 \%)$ and classified as pT3 $(n=58$, $59.2 \%)$. Lymph node metastases were found in 51 patients $(52 \%)$. Therefore, most tumors were classified as stage II ( $n=44,44.9 \%)$ or III $(n=37,37.8 \%)$ according to the UICC classification. The median number of examined lymph nodes was 11(1-33) with a median of $1(0-32)$ tumor-positive lymph node(s). Further characteristics are presented in Table 2.

\section{Statistical Analyses}

Data are reported as frequencies, means, and/or median with percentages. Continuous variables were compared by using the $T$-test, and the chi-square test was used for comparison of categorical variables. Survival and recurrence rates were calculated by the Kaplan-Meier method and compared using the log-rank test. Prognostic factors for survival and recurrence were calculated by using univariate and multivariate Cox regression analyses. Multivariate Cox regression was performed by incorporating as covariates those factors that had $P$-value $\leq 0.1$ on univariate Cox regression analysis.

In total, 50 classifications were generated from 0.0 to $5.0 \mathrm{~mm}$ in steps of $0.1 \mathrm{~mm}$ per class. The optimal limit for the CRMs (in tenths of millimeters) regarding outcome (survival and local recurrence) was calculated for each generated classification with a receiver operating characteristic (ROC) curve, with area under the curve (AUC)
TABLE 1 Patient and tumor characteristics

\begin{tabular}{|c|c|}
\hline Characteristic & $N(\%)$ \\
\hline \multicolumn{2}{|l|}{ Gender } \\
\hline Male & $76(77.6)$ \\
\hline Female & $22(22.4)$ \\
\hline \multicolumn{2}{|l|}{ Age (years) } \\
\hline Median (range) & $65.1(41.4-81.8)$ \\
\hline \multicolumn{2}{|l|}{ Localization } \\
\hline High/mid & $11(11.2)$ \\
\hline Distal & $43(43.9)$ \\
\hline GEJ & $44(44.9)$ \\
\hline \multicolumn{2}{|l|}{ Type of resection } \\
\hline Left transthoracic & $40(40.8)$ \\
\hline Right transthoracic & $58(59.2)$ \\
\hline \multicolumn{2}{|l|}{ Site of anastomosis } \\
\hline Cervical & $54(55.1)$ \\
\hline Intrathoracic & $44(44.9)$ \\
\hline \multicolumn{2}{|l|}{ Histology } \\
\hline Adenocarcinoma & $75(76.5)$ \\
\hline Squamous cell carcinoma & $22(22.4)$ \\
\hline Adeno/squamous cell carcinoma & $1(1.0)$ \\
\hline \multicolumn{2}{|l|}{ Tumor grade } \\
\hline G1 & $9(9.2)$ \\
\hline G2 & $44(44.9)$ \\
\hline G3 & $45(45.9)$ \\
\hline \multicolumn{2}{|l|}{ Tumor stage } \\
\hline I & $15(15.3)$ \\
\hline IIa & $31(31.6)$ \\
\hline $\mathrm{IIb}$ & $13(13.3)$ \\
\hline III & $37(37.8)$ \\
\hline IV & $2(2.0)$ \\
\hline
\end{tabular}

GEJ gastroesophageal junction

analysis and Kaplan-Meier and Cox regression analysis for survival and recurrence rate.

Univariate and multivariate logistic and linear regression analysis were used for calculating variables influencing CRM extension, with CRM as a continuous variable in millimeters. A $P$-value of $<0.05$ was considered to be significant. Statistical analysis was performed by using the statistical package SPSS version 16.0 (SPSS Inc., Chicago, IL).

\section{RESULTS}

\section{Measurement of Circumferential Resection Margins}

The measured CRMs ranged from $0.0 \mathrm{~mm}$ to $>10.0 \mathrm{~mm}$. Twenty-five patients $(25.5 \%)$ had tumor cells to the outer margins (CRM of $0.0 \mathrm{~mm}$ ). Most of these patients $(n=24,96 \%)$ had advanced pT status (pT3/4) 
TABLE 2 Study measurements

\begin{tabular}{ll}
\hline Study characteristics & $N(\%)$ \\
\hline Circumferential margins & \\
Free of tumor & $73(74.5)$ \\
Tumor ingrowth $(0 \mathrm{~mm})$ & $25(25.5)$ \\
Circumferential margins $(\mathrm{mm})$ & \\
$\quad$ Median & 1.0 \\
Mean & 2.0 \\
Range & $0.0-10.0$ \\
Tumor growth & \\
$\quad$ Spray & $59(60.2)$ \\
Pushing & $39(39.8)$ \\
Invasive growth & \\
Lymph vascular space & $39(39.8))$ \\
Perineural & $25(25.5)$ \\
Tumor length (endoscopy), cm & \\
$\leq 5$ & $70(71.4)$ \\
$>5$ & $28(28.6)$ \\
Median (range) & $4.5(0-14)$ \\
Median nodal yield (range) & \\
Examined number & $11(1-33)$ \\
Positive (malignant) number & $1(0-32)$ \\
Positive lymph nodes & \\
$\leq 4$ & $89(72.7)$ \\
Lymph node ratio & $29(29.6)$ \\
$\leq 0.20$ & \\
$>0.20$ & \\
\hline & \\
& \\
&
\end{tabular}

$(P=0.029)$ or had lymph node metastases $(n=18,72 \%$; $P=0.021)$. Staging was therefore unfavorable in patients with CRM of $0 \mathrm{~mm}(P=0.001)$.

\section{Outcome in the Study Group: Recurrent Disease and Survival}

Mean follow-up time was 37 months with a range of 4.7-124 months. In the follow-up period 44 patients $(44.9 \%)$ died due to oncological reasons.

Locoregional recurrence of disease occurred in $46 \%$ of patients within a median period of 15.3 (3.7-78.7) months. Year of surgery $(P=0.2)$, type of surgery $(P=0.83)$, and type of histology (adeno/squamous) $(P=0.544)$ were not associated with development of local recurrence. Recurrent disease occurred more and developed earlier in patients with CRM of $0 \mathrm{~mm}(P<0.001)$, in patients with higher pT status $(P<0.001)$, and in patients with lymph node metastasis $(P<0.001)$. Prognostic factors for recurrent disease on univariate analysis are listed in Table 3. Independent prognostic factors for development of local recurrence as calculated by multivariate analysis were CRM of $0 \mathrm{~mm}$
$(P=0.024)$ and $\mathrm{pN} 1$ status $(P=0.021)$. Although significant on univariate analysis $(P=0.031)$, pT status was not significant $(P=0.29)$ on multivariate analysis.

Overall 5-year survival was $47 \%$. Year of surgery $(P=0.611)$, type of surgery $(P=0.847)$, and type of histology (adeno or squamous) $(P=0.879)$ were not related to survival. Patients with CRM of $0 \mathrm{~mm}$ had significantly worse 5 -year survival $(24 \%$ versus $57 \%$, $P=0.001)$. Several other prognostic factors for survival are listed in Table 3. Independent prognostic factors for survival were presence of recurrent disease $(P<0.001)$, pN1 status $(P=0.025)$, and CRM of $0 \mathrm{~mm}(P=0.05)$.

\section{Cutoff Point of CRMs}

The receiver operating characteristic (ROC) curves for survival and recurrent disease showed the $\leq 1.0 \mathrm{~mm}$ and $>1.0 \mathrm{~mm}$ classification to be optimal among all ROC curves for the 50 subclassifications in tenths of millimeters. Furthermore, survival in patients with $\mathrm{CRM} \leq 1.0 \mathrm{~mm}$ and $>0 \mathrm{~mm}$ did not differ significantly from survival in patients with CRM of $0 \mathrm{~mm}(P=0.43)$. Both had significantly worse outcome than those with CRM $>1.0 \mathrm{~mm}$ ( $P<0.001$ and $P=0.004$, respectively) (Fig. 4). Further comparison of this cutoff point showed that survival was worst in the CRM $\leq 1.0 \mathrm{~mm}$ group versus CRM $>1.0 \mathrm{~mm}$ $(P<0.001)$ independent of all other classifications.

Local recurrence occurred more frequently in patients with CRM $\leq 1.0 \mathrm{~mm}$, with 32 versus 13 patients $(P=0.001)$. Also, time to development of local recurrence was significantly shorter in patients with CRM $\leq 1.0 \mathrm{~mm}$ $(P=0.002)$.

With the cutoff point determined as CRM $\leq 1.0 \mathrm{~mm}$ (CRM+; 47 patients) with an optimal CRM limit of $>1.0 \mathrm{~mm}$ (CRM-; 51 patients), the rate of CRM involvement was $48 \%$.

\section{Division in $C R M+$ and $C R M-$}

Localization of tumor $(P=0.22)$, type of histology $(P=0.77)$, type of resection $(P=0.78)$, location of anastomosis $(P=0.443)$, and year of surgery $(P=0.13)$ were not significantly different in the CRM $>1.0 \mathrm{~mm}$ (CRM-) and CRM $\leq 1.0 \mathrm{~mm}(\mathrm{CRM}+)$ groups. Tumor characteristics as growth pattern (spray or pushing) and grade were comparable in the two groups $(P=0.077$ and $P=0.309)$. Lymph vascular space involvement occurred more frequently in the CRM+ group, being $54 \%(n=28)$ versus $23 \%(n=11)$, respectively $(P=0.002)$. Perineural invasion was equally divided $(P=0.358)$, as was length of tumor $>5 \mathrm{~cm}$ in the lumen of the esophagus $(P=0.525)$.

Regarding pT status, the CRM+ group had a higher number of pT3 tumors $(n=43 ; 84 \%)$ than did the CRM- 
TABLE 3 Prognostic factors for survival and local recurrence of disease: univariate Cox regression analysis

\begin{tabular}{|c|c|c|c|c|}
\hline \multirow[t]{2}{*}{ Factor } & \multirow[t]{2}{*}{ Hazard ratio } & \multicolumn{2}{|c|}{ 95\% Confidential interval } & \multirow[t]{2}{*}{$P$ value } \\
\hline & & Lower & Upper & \\
\hline \multicolumn{5}{|l|}{ Survival } \\
\hline Tumor grade & 1.001 & 0.654 & 1.532 & 0.997 \\
\hline Type of growth & 0.824 & 0.446 & 1.525 & 0.539 \\
\hline Perineural invasion & 1.636 & 0.847 & 3.160 & 0.143 \\
\hline Lymph vascular space involvement & 1.984 & 1.082 & 3.639 & $\mathbf{0 . 0 2 7}$ \\
\hline \multicolumn{5}{|l|}{ Stage } \\
\hline pT stage & 1.338 & 1.048 & 1.707 & 0.019 \\
\hline $\mathrm{pN}$ stage & 5.344 & 2.548 & 11.209 & $<\mathbf{0 . 0 0 1}$ \\
\hline pM1a stage & 3.951 & 1.202 & 12.986 & 0.024 \\
\hline $\mathrm{CRM}$ in $\mathrm{mm}$ & 0.762 & 0.630 & 0.921 & 0.005 \\
\hline CRM ingrowth $(0 \mathrm{~mm})$ & 0.357 & 0.193 & 0.659 & 0.001 \\
\hline Recurrent disease & 9.768 & 4.427 & 21.631 & $<\mathbf{0 . 0 0 1}$ \\
\hline$>4$ positive lymph nodes & 4.904 & 2.520 & 9.564 & $<\mathbf{0 . 0 0 1}$ \\
\hline Lymph node ratio $>0.20$ & 3.987 & 2.164 & 7.346 & $<\mathbf{0 . 0 0 1}$ \\
\hline Tumor length $(\mathrm{cm})$ & 1.120 & 1.006 & 1.248 & $\mathbf{0 . 0 3 9}$ \\
\hline \multicolumn{5}{|l|}{ Local recurrence } \\
\hline Tumor grade & 0.967 & 0.632 & 1.479 & 0.876 \\
\hline Type of growth & 0.812 & 0.441 & 1.496 & 0.504 \\
\hline Perineural invasion & 2.620 & 1.429 & 4.802 & 0.002 \\
\hline Lymph vascular space involvement & 2.412 & 1.327 & 4.383 & 0.004 \\
\hline \multicolumn{5}{|l|}{ Stage } \\
\hline pT stage & 1.304 & 1.025 & 1.660 & $\mathbf{0 . 0 3 1}$ \\
\hline $\mathrm{pN}$ stage & 5.357 & 2.634 & 10.859 & $<\mathbf{0 . 0 0 1}$ \\
\hline pM1a stage & 2.709 & 0.646 & 11.367 & 0.173 \\
\hline $\mathrm{CRM}$ in $\mathrm{mm}$ & 0.754 & 0.623 & 0.913 & 0.004 \\
\hline CRM ingrowth $(0 \mathrm{~mm})$ & 0.358 & 0.198 & 0.648 & 0.001 \\
\hline$>4$ positive lymph nodes & 6.276 & 3.299 & 11.937 & $<\mathbf{0 . 0 0 1}$ \\
\hline Lymph node ratio $>0.20$ & 5.237 & 2.883 & 9.514 & $<\mathbf{0 . 0 0 1}$ \\
\hline Tumor length $(\mathrm{cm})$ & 1.157 & 1.044 & 1.282 & 0.005 \\
\hline
\end{tabular}

Cancer-Specific

Survival (Percent)

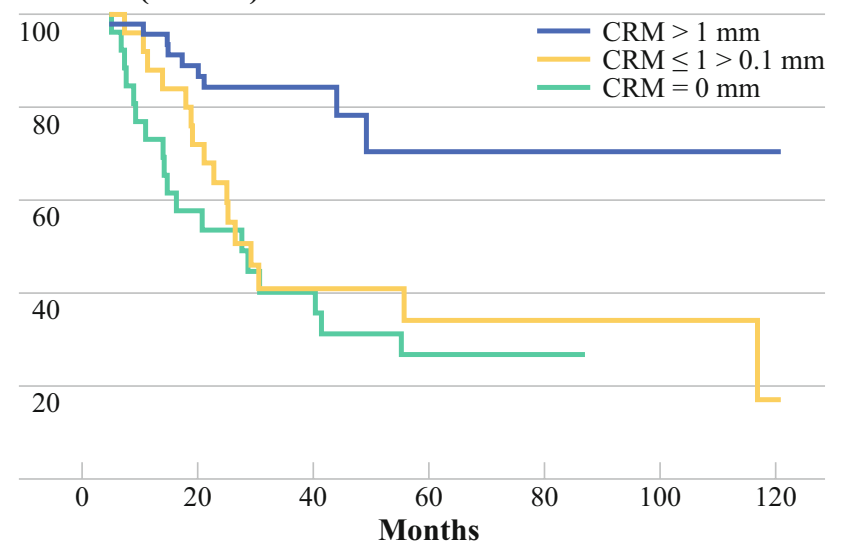

FIG. 4 Cancer-specific survival by CRM in three categories: CRMs with tumor ingrowth $(0 \mathrm{~mm})$ had similar outcome to CRMs $\leq 1 \mathrm{~mm}$ and $>0 \mathrm{~mm}(P=0.43)$; both had significantly worse outcome compared with $\mathrm{CRM}>1 \mathrm{~mm} \quad(P<0.001$ and $P=0.004$, respectively) group ( $n=15 ; 32 \%: P=0.005)$. Also, the rate of $\mathrm{pN} 1$ status was higher in the CRM+ group, being $63 \%(n=32)$ versus $36 \%(n=17)$, respectively $(P=0.015)$, both leading to a significant higher number of stage III tumors in the CRM+ group. However, compared with pT status $(P=0.09)$ and $\mathrm{pN}$ status $(P<0.001), \mathrm{CRM}$ classification $(\leq 1.0 \mathrm{~mm}$ and $>1.0 \mathrm{~mm})$ was an independent prognostic factor for survival $(P=0.011)$. Also, for the development of local recurrence, pN status $(P<0.001)$ and CRM classification $(P=0.042)$ were independent prognostic factors. This CRM classification is therefore a stronger prognostic factor for both survival and development of recurrent disease than is $\mathrm{pT}$ status.

\section{Prognostic Factors for More Extensive CRM Involvement}

Several factors had an impact on the extension of circumferential margins in millimeters, including tumor stage, 
TABLE 4 Prognostic factors for extension of circumferential resection margins involvement, with CRM as continuous variable in mm: logistic and linear regression univariate analysis

\begin{tabular}{lcrrr}
\hline Factor & Regression coefficient & \multicolumn{2}{l}{$95 \%$ Confidential interval } & \multirow{2}{*}{$P$ value } \\
\cline { 3 - 4 } & & Lower & Upper & \\
\hline Year of surgery & 0.042 & -0.167 & 0.251 & 0.693 \\
Type of resection & -0.008 & -0.054 & 0.038 & 0.737 \\
Histology type & -0.027 & -0.099 & 0.045 & 0.465 \\
Localization & -0.015 & -0.083 & 0.054 & 0.666 \\
Tumor grade & -0.031 & -0.085 & 0.022 & 0.251 \\
Stage & -0.272 & -0.358 & -0.186 & $<\mathbf{0 . 0 0 1}$ \\
pT stage & -0.110 & -0.186 & -0.034 & $\mathbf{0 . 0 0 5}$ \\
pN stage & -0.073 & -0.112 & -0.033 & $<\mathbf{0 . 0 0 1}$ \\
pM stage & -0.010 & -0.024 & 0.005 & 0.187 \\
Tumor growth (spray) & 0.052 & 0.012 & 0.092 & $\mathbf{0 . 0 1 1}$ \\
Perineural invasion & -0.036 & -0.072 & 0.000 & $\mathbf{0 . 0 4 8}$ \\
Lymph vascular space involvement & -0.078 & -0.116 & -0.040 & $<\mathbf{0 . 0 0 1}$ \\
$>4$ positive lymph nodes & -0.040 & -0.071 & -0.010 & $\mathbf{0 . 0 1 0}$ \\
Lymph node ratio $>0.20$ & -0.047 & -0.084 & -0.010 & $\mathbf{0 . 0 1 4}$ \\
Tumor length (cm) & -0.370 & -0.579 & 0.160 & $\mathbf{0 . 0 0 1}$ \\
\hline
\end{tabular}

depth of tumor ingrowth according to pT stage, and occurrence of lymph node metastasis. These prognostic factors influencing the extent of CRM involvement are listed in Table 4. Independent prognostic factors for CRM involvement were satellite growth of the tumor $(P=0.036)$, pT stage $(P=0.019)$, and tumor size $>5 \mathrm{~cm}$ $(P=0.01)$.

\section{Resection Margins Versus CRMs}

CRM - patients had significant longer survival and fewer events than CRM+ patients and patients in the control group with an $\mathrm{R} 1$ resection $(P=0.002$ and $P<0.001$, respectively). There was no statistically difference in survival between CRM+ patients and patients of the control group $(P=0.12)$ (Fig. 5).

There were significantly more cancer-related deaths during follow-up in the CRM+ and R1 groups than in the CRM- group, being 66.6\% $(n=34)$ and 90\% $(n=19)$, respectively, versus $21 \%(n=10)(P<0.001)$.

\section{DISCUSSION}

In the assessment of surgical radicality in esophageal cancer, circumferential resection margin (CRM) is an independent prognostic factor for both local recurrence and survival. The clinical importance of CRM in current histopathologic staging procedure is considerably underestimated. Patients with CRM involvement have worse outcome, independent of other factors. We determined that the best cutoff point for CRM was $\leq 1.0 \mathrm{~mm}$ and $>1.0 \mathrm{~mm}$. A tumor-free CRM limit $>1.0 \mathrm{~mm}$ seems optimal regarding its

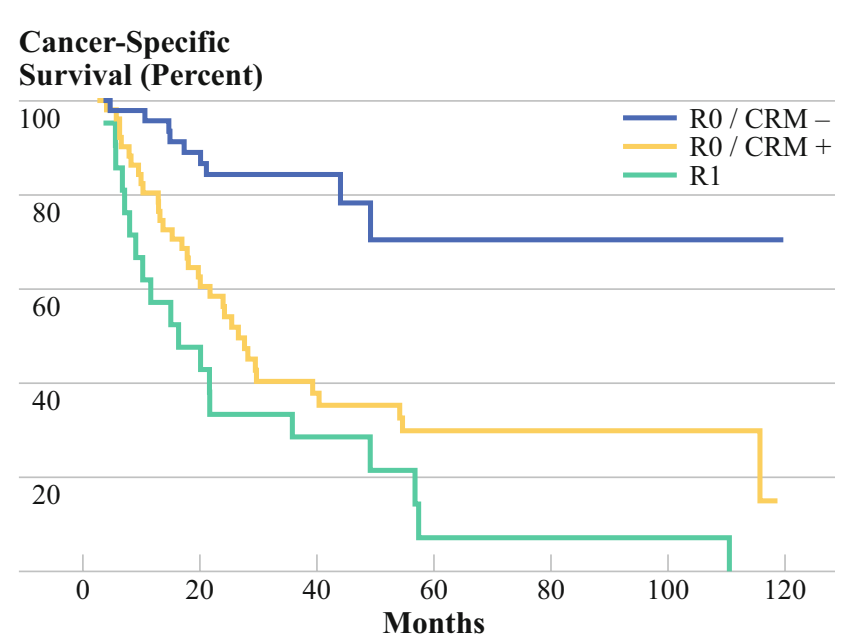

FIG. 5 Equal cancer-specific survival of positive CRMs and R1 resections. Kaplan-Meier survival curve of patients with positive resection margins (R1) of the control group $(n=21)$ and patients of the study group with positive CRM $\leq 1 \mathrm{~mm}(\mathrm{CRM}+)(n=51,52 \%)$ and free $\mathrm{CRM}>1 \mathrm{~mm}(\mathrm{CRM}-)(n=47,48 \%)$. There was no significant difference in survival between CRM+ and R1 $(P=0.12)$; significant differences were found between CRM - with $\mathrm{CRM}+$ and R1 (both $P<0.001$ )

prognostic value for development of recurrent disease and survival. The rate of CRM involvement $(\leq 1.0 \mathrm{~mm})$ in this study was $48 \%$, which is comparable to figures from other studies. ${ }^{14}$ Patients with CRM $\leq 1.0 \mathrm{~mm}$ had equal survival compared with patients with an $\mathrm{R} 1$ resection.

Total lymph node count and lymph node ratio in this study are in line with the results of other large, single-center studies. ${ }^{19,20}$ Although lymph node involvement occurred more frequently in patients with $\mathrm{CRM} \leq 1.0 \mathrm{~mm}$, it had no influence on the independent prognostic factor of CRM. 
CRM classification into $\leq 1.0 \mathrm{~mm}$ and $>1.0 \mathrm{~mm}$ is also a stronger prognostic factor for survival and local recurrence than is pT status of the tumor.

We reappraised the prognostic value of several histopathologic measurements and can affirm the prognostic importance of lymph vascular space involvement and perineural invasion for survival and local recurrence. ${ }^{16-18,20-22}$ The used classifications of $>4$ positive lymph nodes and lymph node ratio $>0.20$ were highly significant for survival and recurrent disease and also correlated with extensive CRM involvement on univariate analysis. Our data support published reports on their usefulness in the estimation of prognosis for survival and add their prognostic value for CRM involvement. ${ }^{16,23}$

Independent factors for extensive CRM involvement were satellite (spray-type) growth of tumor, depth of tumor ingrowth according to pT classification, and length of tumor.

Other studies defined CRM involvement in esophageal cancer as presence of tumor within $1 \mathrm{~mm}$ of inked margins, while no explanation is given for this choice of cutoff point. We assume that this choice is based on the $\mathrm{R}$ classification of $1 \mathrm{~mm}$ and/or the cutoff point for CRMs in rectal cancer of $\leq 1.0 \mathrm{~mm}$. In this study we found that margins $\leq 1.0 \mathrm{~mm}$ and $>0 \mathrm{~mm}$ and margins with obvious tumor ingrowth $(0 \mathrm{~mm})$ did not have different survival and had the same prognostic value for dismal prognosis. The receiver operating characteristic (ROC) curve and survival analysis demonstrated the best sensitivity and specificity for the $\leq 1.0 \mathrm{~mm}$ and $>1.0 \mathrm{~mm}$ classification out of 50 subclassifications in tenths of millimeters. Therefore, the optimal limit of CRM involvement and its predictive value for outcome should be $>1.0 \mathrm{~mm}$.

As in rectal cancer, patients with esophageal carcinoma and CRM involvement are more likely to develop local recurrence, while local recurrence of disease is the strongest predictor for dismal outcome. ${ }^{8}$

Considerable numbers of studies have been published regarding prognostic factors for esophageal carcinoma. However, only a few studies included in-depth analyses of CRM involvement and homogeneous study groups. The major advantage of our study is that we report CRM results from a statistically comparable group of patients operated on at a single center, with the same referral pattern, selection process, classification procedure, resection procedure, and follow-up protocol. Our data are not affected by neoadjuvant chemoradiation or differences in surgical procedure.

Extension of CRM is correlated with more advanced pT status; perhaps other studies therefore lack consistent CRM measurements. However, tumor depth (pT) is only related to extent of tumor ingrowth in layers, whereas CRMs may also depend on differences in cellular layer thickness of the esophageal wall and the performed surgical technique of resection. For example, unexpected ruptures or incomplete resection may occur during surgery, particularly when stretching or dissecting the esophagus from the periesophageal tissue during transhiatal procedures (Fig. 3). ${ }^{13}$ Therefore it is plausible that adequately performed extended transthoracic resection reduces the likelihood of involvement of CRMs and this may partly explain the improved survival in the randomized study of Hulscher et al. ${ }^{24}$ Moreover, it explains the independent prognostic factor for development of recurrent disease and survival of CRM involvement, independent of $\mathrm{pT}$ and $\mathrm{pN}$ classification. In advocating a surgical procedure, the ideal control group to differentiate pattern of recurrence (local versus recurrence outside the conduit) and survival would consist of patients who underwent a non-en bloc procedure (i.e., transhiatal resection) with a positive circumferential margin. Hence, CRM measurements can serve as a useful quality indicator of surgery.

As the proposed CRM $>1 \mathrm{~mm}$ is not always feasible, even with an en bloc transthoracic resection, neoadjuvant chemoradiation can play an important role in increasing the ratio of clear CRMs. In this study no patients underwent study-related neoadjuvant treatment. Therefore no data on the effect of neoadjuvant treatment on CRM involvement can be given. However, recent studies showed that neoadjuvant multimodality treatment significantly reduces CRM involvement. $5,25,26$ With the worse prognosis of CRM involvement in mind, this could signify an important benefit of neoadjuvant treatment and might even explain in part the improved results of this type of therapy. Since most patients with esophageal cancer currently receive induction therapies, CRM measurements could be used as a marker of tumor regression after neoadjuvant treatment. In rectal cancer, CRM measurements after neoadjuvant therapy can predict local recurrence and a subsequently worsened outcome. ${ }^{10}$

In recent years many studies have suggested an adaptation of the TNM staging system with assimilation of many different, scientifically proven prognostic factors. ${ }^{17,20,22,23,27}$ With increasing knowledge about the biological behavior and findings of strong prognostic variables other than TNM, adaptation of the staging procedure will be inevitable in the near future. We suggest that CRM measurement should be a part of the classification, because it seems to have more value than pT status alone. At least integration of CRM measurement with the $\mathrm{R}$ classification is pivotal. We propose that the resection $(\mathrm{R})$ classification should not only include clear longitudinal margins but also circumferential resection margins with a critical limit of $1.0 \mathrm{~mm}$.

In conclusion, we advocate integration of the circumferential resection margin $(\mathrm{CRM})$ with a limit of $\leq 1.0 \mathrm{~mm}$ and $>1.0 \mathrm{~mm}$ into the routine pathologic staging procedure 
of esophageal carcinoma. CRM is an important underestimated independent prognostic factor for development of recurrent disease and survival. Therefore, patients with unfavorable CRM involvement should be approached as patients with an R1 resection with corresponding outcome. Furthermore, we recommend radical transthoracic extended resection to achieve optimal surgical margins, including ample circumferential resection margins, for better locoregional control and long-term outcome.

ACKNOWLEDGMENT We thank G. A. Kock, MD, for his helpful support with the start of the CRM measuring process.

OPEN ACCESS This article is distributed under the terms of the Creative Commons Attribution Noncommercial License which permits any noncommercial use, distribution, and reproduction in any medium, provided the original author(s) and source are credited.

\section{REFERENCES}

1. Powell J. Continuing rising trend in oesophageal adenocarcinoma. Int J Cancer. 2002;102:422-7.

2. Plukker JT, van Westreenen HL. Staging in oesophageal cancer. Best Pract Res Clin Gastroenterol. 2006;20:877-91.

3. Lerut T, Coosemans W, De Leyn P, Decker G, Deneffe G, Van Raemdonck D. Is there a role for radical esophagectomy. Eur $J$ Cardiothorac Surg. 1999;16 Suppl 1:S44-S47.

4. Shao LF, Gao ZG, Yang NP, Wei GQ, Wang YD, Cheng CP. Results of surgical treatment in 6,123 cases of carcinoma of the esophagus and gastric cardia. J Surg Oncol. 1989;42:170-4.

5. Mulligan ED, Dunne B, Griffin M, Keeling N, Reynolds JV. Margin involvement and outcome in oesophageal carcinoma: a 10-year experience in a specialist unit. Eur J Surg Oncol. 2004:30:313-7.

6. Gall CA, Rieger NA, Wattchow DA. Positive proximal resection margins after resection for carcinoma of the oesophagus and stomach: effect on survival and symptom recurrence. Aust NZ J Surg. 1996;66:734-7.

7. Birbeck KF, Macklin CP, Tiffin NJ, Parsons W, Dixon MF, Mapstone NP, et al. Rates of circumferential resection margin involvement vary between surgeons and predict outcomes in rectal cancer surgery. Ann Surg. 2002;235:449-57.

8. Adam IJ, Mohamdee MO, Martin IG, Scott N, Finan PJ, Johnston $\mathrm{D}$, et al. Role of circumferential margin involvement in the local recurrence of rectal cancer. Lancet. 1994;344:707-11.

9. Luna-Perez P, Bustos-Cholico E, Alvarado I, Maffuz A, Rodriguez-Ramirez S, De La Barrera MG, et al. Prognostic significance of circumferential margin involvement in rectal adenocarcinoma treated with preoperative chemoradiotherapy and low anterior resection. J Surg Oncol. 2005;90:20-5.

10. Nagtegaal ID, Quirke P. What is the role for the circumferential margin in the modern treatment of rectal cancer? J Clin Oncol. 2008;26:303-12.

11. Heald RJ, Ryall RD. Recurrence and survival after total mesorectal excision for rectal cancer. Lancet. 1986;1:1479-82.
12. Sagar PM. Significance of circumferential resection margin involvement after oesophagectomy for cancer. $B r J$ Surg. 1993;80:1386-8.

13. Kuwano H, Masuda N, Kato H, Sugimachi K. The subepithelial extension of esophageal carcinoma for determining the resection margin during esophagectomy: a serial histopathologic investigation. Surgery. 2002;131:S14-S21.

14. Dexter SP, Sue-Ling H, McMahon MJ, Quirke P, Mapstone N, Martin IG. Circumferential resection margin involvement: an independent predictor of survival following surgery for oesophageal cancer. Gut. 2001;48:667-70.

15. Sobin LH. TNM, sixth edition: new developments in general concepts and rules. Semin Surg Oncol. 2003;21:19-22.

16. Mariette C, Piessen G, Briez N, Triboulet JP. The number of metastatic lymph nodes and the ratio between metastatic and examined lymph nodes are independent prognostic factors in esophageal cancer regardless of neoadjuvant chemoradiation or lymphadenectomy extent. Ann Surg. 2008;247:365-71.

17. Bogoevski D, Onken F, Koenig A, Kaifi JT, Schurr P, Sauter G, et al. Is it time for a new TNM classification in esophageal carcinoma? Ann Surg. 2008;247:633-41.

18. Heeren PA, van Westreenen HL, Geersing GJ, van Dullemen HM, Plukker JT. Influence of tumor characteristics on the accuracy of endoscopic ultrasonography in staging cancer of the esophagus and esophagogastric junction. Endoscopy. 2004;36: 966-71.

19. Mariette C. Extent of oesophageal resection for adenocarcinoma of the oesophagogastric junction. Eur J Surg Oncol. 2003;29: 588-93.

20. Bollschweiler E, Baldus SE, Schroder W, Schneider PM, Holscher AH. Staging of esophageal carcinoma: length of tumor and number of involved regional lymph nodes. Are these independent prognostic factors? J Surg Oncol. 2006;94:355-63.

21. Eloubeidi MA. Prognostic factors for the survival of patients with esophageal carcinoma in the U.S.: the importance of tumor length and lymph node status. Cancer. 2002;95:1434-43.

22. Griffiths EA, Brummell Z, Gorthi G, Pritchard SA, Welch IM. Tumor length as a prognostic factor in esophageal malignancy: univariate and multivariate survival analyses. J Surg Oncol. 2006;93:258-67.

23. Wijnhoven BP, Tran KT, Esterman A, Watson DI, Tilanus HW. An evaluation of prognostic factors and tumor staging of resected carcinoma of the esophagus. Ann Surg. 2007;245:717-25.

24. Hulscher JB, van Sandick JW, de Boer AG, Wijnhoven BP, Tijssen JG, Fockens $P$, et al. Extended transthoracic resection compared with limited transhiatal resection for adenocarcinoma of the esophagus. $N$ Engl J Med. 2002;347:1662-9.

25. Sujendran V, Wheeler J, Baron R, Warren BF, Maynard N. Effect of neoadjuvant chemotherapy on circumferential margin positivity and its impact on prognosis in patients with resectable oesophageal cancer. Br J Surg. 2008;95:191-4.

26. Saha AK, Sutton C, Rotimi O, Dexter S, Sue-Ling H, Sarela AI. Neoadjuvant chemotherapy and surgery for esophageal adenocarcinoma: prognostic value of circumferential resection margin and stratification of N1 category. Ann Surg Oncol. 2009;16:136470.

27. Lagarde SM, Ten Kate FJ, Reitsma JB, Busch OR, van Lanschot JJ. Prognostic factors in adenocarcinoma of the esophagus or gastroesophageal junction. J Clin Oncol. 2006;24:4347-55. 